\title{
Dynamic Programming Based Link Cost Function and Load Estimation Method for ATM Links Applying Routing Intelligence in ATM Networks
}

\author{
M. Conte \\ Siemens AG Österreich \\ Boschstraße 10, 1190 Wien, Austria \\ marco.conte@siemens.at
}

Key words: Dynamic Routing, Dynamic Programming, ATM

\begin{abstract}
In connection-oriented networks dynamic routing schemes are designed to improve the call blocking performance by introducing network intelligence. An accurate model describing a networks statistical behaviour is the network Markov model. Based on this model the theory of dynamic programming for Markov processes (also called the Markov Decision Process theory, MDP) has in the past successfully been applied in the field of dynamic routing. The Forward Looking Routing (FLR) scheme [1] defines a dynamic link cost function for single-rate circuit-switched networks. In ATM networks carrying multirate traffic a more complex traffic theory is encountered than for singlerate traffic. Applications of the MDP concept for routing multirate traffic are made for example in [2], [3], [4] and [5]. In this work, a state dependent link cost function for multirate links is derived, based on the scalar link state model introduced in [5]. It is shown that for the single rate traffic model, this link cost function becomes equal to the FLR link cost function. An approximation method is proposed for determining the offered link load in weak mesh networks. Furthermore, a scheme for estimating the temporal evolution of the carried link load is developed. It is shown by simulation how the ATM link cost function and load estimation may be used within the frame of a dynamic routing scheme called DR/ATM. The performance of the DR/ATM scheme in simulation runs involving different network topologies is presented. Moreover the performance of the ATM link cost function in combination with link state flooding is shown. The simulation runs show that the blocking performance is substantially improved as compared with standard reference routing schemes.
\end{abstract}

The original version of this chapter was revised: The copyright line was incorrect. This has been corrected. The Erratum to this chapter is available at DOI: 10.1007/978-0-387-35522-1_37 


\section{INTRODUCTION}

Routing in communication networks consists of finding paths, consisting of one or several links, through the network. The routing problem arises in connection-oriented telephony networks as well as in connection-less data networks. For telephony networks it is well known that the quality of the routing scheme employed significantly influences the call blocking performance, as shown e.g. in [1]. The Asynchronous Transfer Mode (ATM) supports connections with different and even variable bit rates and thus it allows to construct shared voice and data networks. Recent simulation results show that dynamic routing schemes generally reduce the blocking probability, or equivalently increase the throughput at a given blocking level, in multirate ATM networks, by introducing some additional network intelligence. For the design of a dynamic ATM routing scheme a trade-off between the benefits of routing optimisation and computational complexity must be carried out.

The present results were obtained as a part of a research study on dynamic routing which was carried out at Siemens Austria, under European Space Agency contract. The work started in 1996 with the scope of identifying promising dynamic routing scenarios for future ATM core networks and to analyse the possibility of involving satellite transmission to distribute routing related data. Some elements of dynamic routing for circuit switched telephony networks were reconsidered within an ATM environment. In particular the estimation of actual link loads from the frequency of call rejections was analysed. This technique was also used in a previous study [6]. A model dynamic routing scheme, called DR/ATM, was developed. DR/ATM was first developed for fully meshed networks, however, the scheme was later adapted to work within weak mesh networks as well. Finally, the ATM link cost functions were derived, which will mainly be discussed in the following.

\section{ATM TRAFFIC THEORETICAL ASPECTS}

\subsection{Theoretical Background}

ATM connections may specify individual bandwidth requirements in their traffic contract, depending of the connection type. Within an ATM network, an ATM node may receive a connection request to another node at any time. Consider short time intervals, where the number of existing 
connections is observed. Call requests arrive randomly, with the same constant probability for each time interval. This probability is independent of any previous call request. Existing connections may be terminated in each of the time intervals, with constant probability, independently of other calls. These assumptions are made, when call inter-arrival times, as well as call holding times of ATM connections are modelled as independent, exponentially distributed random values. This traffic model is also called Poisson-traffic model. The following derivations are based on the Poissontraffic model. The carried link load of an ATM link carrying Poissonian ATM traffic is described as a vector $\underline{x}=\left(x_{1}, \ldots, x_{K}\right)$, where $x_{i}$ is the number of established connections of type $i, i=1, \ldots, K . K$ is the number of connection request types which can occur. The vector $\underline{x}$ is also called the $K$-dimensional link state. The requested bandwidths of type $i$ calls are denoted as $c_{i}, i=1$, $\ldots, K, c_{1} \leq c_{2} \leq \ldots c_{K}$. The space of possible link states is delimited by the capacity of the link $C$ as follows:

$$
\underline{c} \cdot \underline{x}=\sum_{i=1}^{K} c_{i} x_{i} \leq C, \text { and } x_{i} \geq 0, \forall i=1, \ldots, C .
$$

The random process generated by the link states $\underline{x}$ during a period of time is a $K$-dimensional Markov process. I.e., at a given time, the transition probabilities from the actual state of the link $\underline{x}$ to other states do not depend on link states in the past, given the present state $\underline{x}$. More details about multidimensional traffic models can be found e.g. in [7] and [8].

In practical applications it may occur that the exact number of established calls is not known, whereas only the total occupied link capacity $u=\underline{c} \cdot \underline{x}$ is known. Possible values of $u$ are $0,1, \ldots, C$, assuming that all requested bandwidth values $c_{i}, i=1, \ldots, K$ are integers. The scalar $u$ represents a random process in time, referred to as the scalar link state process. Depending on the requested bandwidth values, it may occur that some of the scalar states $0,1, \ldots, K$ are never reached. For example, if all requested bandwidth values are even, no odd scalar link state can ever be reached. A recursive algorithm for the computation of the steady state probabilities $q_{i}, i=1, \ldots, C$, of the scalar link state process is shown in [9]. The computation is as follows:

$$
q_{u}=\frac{1}{u} \sum_{i=1}^{K} c_{i} a_{i} q_{u-i} u=1, \ldots, K, \text { and } q_{0}=\left(\sum_{i=1}^{C} q_{i}\right)^{-1} \text {, }
$$


where $a_{i}=\lambda_{i} \cdot \tau_{i}$ is the offered load, $\lambda_{i}$ is the arrival rate and $\tau_{i}$ is the mean duration for call type $i, i=1, \ldots, K$. In contrast to the $K$-dimensional link state process, the scalar link state process is not a Markov Process, i.e. the transition rates from a state $u$ to other states are not independent of the past given $u$. Nevertheless the process may be treated as a Markov process. This method is also applied in [5]. Another interpretation of this approximation is that a Markov process is defined, which has the same states and steady state probabilities $q_{i}, i=1, \ldots, C$ and, on average, the same transition rates as the aggregated link state process. This process will be called the markovian aggregated link state process. The transition rates $a_{i, j}$ between states $i$ and $j$ of the markovian aggregated link state process are:

$$
\begin{aligned}
& a_{u, u+c_{i}}=\lambda_{i}, \text { if } u+c_{i} \leq C, i=1, \ldots, K \\
& a_{u, u-c_{i}}=\frac{\lambda_{i} q_{u-c_{i}}}{q_{u}}, \text { if } u \geq c_{i}, q_{u}>0, i=1, \ldots, K, \\
& a_{u, w}=0, \text { for all other states } \mathrm{u} \text { and } \mathrm{w}(u \neq w) . \\
& \text { By defining } a_{u, u}=-\sum_{\substack{i=0 \\
i \neq u}}^{C} a_{u i}, u=1, \ldots, C,
\end{aligned}
$$

the matrix of transition rates $A$ of the Markovian aggregated link state process can be written as the $(C+1) \times(C+1)$ square matrix $A=\left(a_{i j}\right)$. For the steady state probability vector $q=\left(q_{0}, q_{1}, \ldots, q_{C}\right)$ the following system of equations is obtained:

$$
\sum_{i=0}^{C} q_{i} a_{i k}=0, \text { for } k=0, \ldots, C,
$$

which can be written as a matrix equation: $\underline{q} A=\underline{0}$, where $\underline{0}$ is the $C+1$ dimensional zero vector. The loss rate $L$ of the Markovian aggregated link state process is obtained as:

$$
L=\sum_{i=1}^{K} \lambda_{i} b_{i}
$$

where $b_{i}$ is the blocking probability for call type $i$. For a complete bandwidth sharing policy among the call types the blocking probabilities can be obtained from the steady state probabilities: 


$$
b_{i}=\sum_{u=C-c_{i}+1}^{C} q_{u}
$$

Thus for complete bandwidth sharing, the blocking rates of the Markovian aggregate link state process are equal to the blocking rates of the original aggregate link state process. According to the theory of dynamic programming, [10], the following system of differential equations is obtained for the expected loss $v_{u}(t)$ at time $t$, given the initial state $u$ :

$$
\frac{d}{d t} v_{u}(t)=r_{u}+\sum_{j=0}^{C} a_{u j} v_{j}(t), u=0, \ldots, C,
$$

where $r_{u}$ is the loss rate in state $u$. For long term considerations, $v_{u}(t)$ may be replaced by the asymptotic linear curve of the expected loss: $L \cdot t+z_{u}$, where the constant $z_{u}$ depends on the initial state $u$ of the link. The system of differential equations (7) becomes then a system of linear equations:

$$
L=r_{u}+\sum_{j=0}^{C} a_{u j} z_{j}, u=0, \ldots, C,
$$

which can be written as a matrix equation: $\underline{L}=\underline{r}+A \underline{z}$, where $\underline{L}$ is the $C+1$-dimensional column vector $(L, \ldots, L)^{T}, \quad \underline{r}=\left(r_{0}, \ldots, r_{C}\right)^{\frac{L}{T}}$ and $z=\left(z_{0}, \ldots, z_{C}\right)^{T}$. The values $z_{i}, i=0,1, \ldots, C$ are called the Howard relative costs. In [5] it is proposed that the system of equations (8) is solved, setting $z_{0}=0$. The reduced Howard relative cost $v_{i j}=z_{j}-z_{i}$ of $j$ with respect to $i$ is then used as link admission criterion.

\subsection{Link Cost Function}

After this overview of traffic theoretical methods we now proceed to the derivation of the multirate link cost function. Before introducing the cost function, we state a first lemma. Let $q_{i}$ be the $C+1$-dimensional vector containing the value $q_{i}$ at the $i$-th position and having all other entries equal to zero, i.e. $y_{i}=\left(0, \ldots, 0, q_{i}, 0, \ldots, 0\right)$. The following equations are obtained using the definition of matrix $A$ : 


$$
\underline{q}_{n} A \underline{z}=-\sum_{\substack{i=0 \\ n-c_{i} \geq 0}}^{K} q_{n-c_{i}} \lambda_{i}\left(z_{n}-z_{n-c_{i}}\right)+\sum_{\substack{i=0 \\ n+c_{i} \leq C}}^{K} q_{n} \lambda_{i}\left(z_{n+c_{i}}-z_{n}\right), n=0,
$$

In both summations the index $i$ is taken from zero to $K$, only over those values of $i$, for which $n-c_{i} \geq 0$, respectively $n+c_{i} \leq C$.

Lemma 1:

$$
\sum_{n=0}^{u} \underline{q}_{n} A \underline{z}=\sum_{\substack{n=u-c_{X}+1, n \geq 0}}^{u} q_{n} \sum_{\substack{i=0 \\ u \leq n+c_{i}<C}}^{K} \lambda_{i}\left(z_{n+c_{i}}-z_{n}\right), n=0,1, \ldots, C .
$$

Proof (by induction): for $u=0$ lemma 1 becomes equal to equation (9), for $n=0$. Assuming that equation (10) is proven for a certain value $u \geq 0$, it is obtained with equation (9) that:

$$
\begin{aligned}
& \sum_{n=0}^{u} \underline{q}_{n} A \underline{z}+\underline{q}_{u+1} A \underline{z}=\sum_{\substack{n=u-c_{K}+1, n \geq 0}}^{u} q_{n} \sum_{\substack{i=0, u \leq n+c_{i}<C}}^{K} \lambda_{i}\left(z_{n+c_{i}}-z_{n}\right)- \\
& -\sum_{\substack{i=0 \\
u+1-c_{i} \geq 0}}^{K} q_{u+1-c_{i}} \lambda_{i}\left(z_{u+1}-z_{u+1-c_{i}}\right)+\sum_{\substack{i=0 \\
u+1+c_{i} \leq C}}^{K} q_{u+1} \lambda_{i}\left(z_{u+1+c_{i}}-z_{u+1}\right)
\end{aligned}
$$

It can be seen that the second sum cancels with the indices $n$ and $i$ of the first (double) sum, for which $n+c_{i}=u+1$, respectively $n=u+1-c_{i}$. Notice hereby that $c_{K}=\max \left\{c_{1}, \ldots, c_{K}\right\}$. The right hand side of (11) now becomes to:

$$
\begin{aligned}
& \sum_{\substack{n=u-c_{K}+2, n \geq 0}}^{u} q_{n} \sum_{\substack{i=0, u+1 \leq n+c_{i}<C}}^{K} \lambda_{i}\left(z_{n+c_{i}}-z_{n}\right)+\sum_{\substack{i=0 \\
u+1+c_{i} \leq C}}^{K} q_{u+1} \lambda_{i}\left(z_{u+1+c_{i}}-z_{u+1}\right)= \\
= & \sum_{\substack{n=(u+1)-c_{K}+1, n \geq 0}}^{u+1} q_{n} \sum_{\substack{i=0, u+1 \leq n+c_{i}<C}}^{K} \lambda_{i}\left(z_{n+c_{i}}-z_{n}\right)=\sum_{\substack{n=(u+1)-c_{K}+1, n \geq 0}}^{u+1} \underline{q}_{n} A z,
\end{aligned}
$$

which proofs the validity of the assumption (10). $\square$ The value $\gamma_{i k}$ is now defined as follows: if a call of type $k$ can be established, starting at state $i, \gamma_{i k}$ is equal to the reduced Howard relative cost of the transition. If starting at $i$ a call of type $k$ is blocked, $\gamma_{i k}$ is equal to 1 . In formulas: 


$$
\gamma_{i k}=\left\{\begin{array}{cl}
z_{i+c_{k}}-z_{i} & , \text { if } i+c_{k} \leq C \\
1 & , \text { if } i+c_{k}>C
\end{array} \forall i=0, \ldots, C, k=1, \ldots, K\right.
$$

Moreover we let $R_{u}$ be the loss rate of the link under complete bandwidth sharing policy, prohibiting states greater than $u$.

$$
R_{u}=\sum_{\substack{i=-c_{K}+1 \\ i \geq 0}}^{K} q_{i} \sum_{\substack{j=0 \\ i+c_{j}>u}}^{K} \lambda_{j}
$$

Lemma 2: The expected value of $\gamma_{i k}$ which occur for transitions from a state $i$ lower than or equal to the state $u$ towards states above $u$, or for call blockings at state $i$ is given by:

$$
E\left[\gamma_{i k} \mid 0 \leq i \leq u, i+c_{k}>u\right]=\frac{\sum_{i=0}^{u} \underline{q}_{i}(A \underline{z}+\underline{r})}{R_{u}}, u=0,1, \ldots, C .
$$

Proof: by means of equation (10) and (14). The probability $P(m \rightarrow n \mid u)$ that, at a call arrival, a transition over the state $u$ occurs from a state $m(u$ $\left.c_{K}<m \leq u, m \geq 0\right)$ to a state $m+c_{n}(1 \leq n \leq K)$ is equal to $q_{m} \lambda_{n} / R_{u}$. For the probability $P($ loss $\mid m)$ for a call loss starting from $m$ we obtain $P($ loss $\mid m)=q_{m} r_{m} / R_{u}$. By the definition of the conditional expectation value we obtain $E\left[\gamma_{i k} \mid 0 \leq i \leq u, i+c_{k}>u\right]=\Sigma P(m \rightarrow n \mid u) \cdot\left(z_{n}-z_{m}\right)+$ $\Sigma P($ loss $\mid m) \cdot 1$ and, by inserting, equation (15) is obtained. $\square$

We may now define the link cost function based on the actual aggregated state $u, \operatorname{cost}(u)=E\left[\gamma_{i k} \mid 0 \leq i \leq u, i+c_{k}>u\right]$, and we define $L_{u}$ as the rate of transitions from a state lower than or equal to $u$ to states greater than $u$ plus the blocking rate from states lower than or equal to $u$, under complete bandwidth at all states $0,1, \ldots, C$. More precisely $L_{u}$ is defined as:

$$
L_{u}=\left(\sum_{i=0}^{u} q_{i}\right)^{-1} R_{u}
$$

We are now ready to state the following theorem: 
Theorem 1: $\operatorname{cost}(u)=\frac{L}{L_{u}}, u=0,1, \ldots, C$,

Proof: from equation (8) we know that $L=A z+\underline{r}$. Thus inserting into equation (15), we deduce cost $(u)=L \cdot \Sigma_{i} q_{i} / R_{u}=L / L_{u} \cdot \square$ If a single call type exists, the ATM link cost function $\operatorname{cost}(u)$ reduces to the well known link cost function for single rate circuit-switched traffic $B(N, A) / B(i, A)$, where $B$ denotes the Erlang loss formula, $N$ is the number of trunks, $A$ is the offered load and $i$ is the actual number of occupied trunks. We call equation (17) the "quotient formula" for multirate traffic and the cost function $\operatorname{cost}(u)$ the ATM-FLR cost function.

\subsection{Determining the Offered Link Load}

The call arrival rate for a link within an ATM network comprise the offered traffic between the start and the end node of the link, the directly carried traffic, as well as traffic which is routed over paths containing several links. For full and strong mesh networks we assumed that the first routing attempt is made on a pre-defined default path. The ATM link cost function is then computed for the default offered load. For weak mesh networks, where no default routes are used, the assumption is made that for a sufficient number of node pairs more than one equivalent minimum-hop paths exist, such that the traffic can be distributed among these paths. As a result, the traffic can be distributed among the links without increasing the sum of all link loads. Assuming optimal traffic distribution, the following optimisation problem is obtained:

$$
\min \sum_{i=1}^{N} L_{(i)}\left(\lambda_{(i)}\right), \text { given that } \sum_{i=1}^{N} \lambda_{(i)}=c
$$

where $N$ is the number of links, $L_{(i)}\left(\lambda_{i i}\right)$ is the loss rate of link $i$, given the total call arrival rate $\lambda_{i}$ on link $i, i=1,2, \ldots, N$. The Lagrange function $\Phi\left(\lambda_{11}, \ldots, \lambda_{(N)}, w\right)$ for $(18)$ is given as:

$$
\Phi\left(\lambda_{(1)}, \ldots, \lambda_{(N)}, w\right)=\sum_{i=1}^{N} L_{(i)}\left(\lambda_{(i)}\right)+w \sum_{i=1}^{N} \lambda_{(i)},
$$

where $w$ is a Lagrange multiplier. By differentiating with respect to $\lambda_{i}$, $i=1, \ldots, N$ the following optimality conditions are obtained: 


$$
\frac{d}{d \lambda_{i}} L_{(i)}\left(\lambda_{(i)}\right)=w, \forall i=1,2, \ldots, N
$$

Equation (20) states that if the assumption of optimal traffic distribution is fulfilled, which may be approximately the case in weak mesh networks, the derivatives of the link loss rates with respect to the total call arrival of the respective link must be equal. We use equation (20) to determine the offered link loads in weak mesh networks.

\subsection{Link Load Estimation}

If the aggregate link state $u_{0}$ at an initial time $t_{0}$ is known, the question arises as to, how the expected link load will evolve after $t_{0}$. As described in [10], for the Markovian aggregate link state process the following system of differential equations is obtained for the state probability distribution at time $t, \underline{\pi}(t)=\left(\pi_{0}(t), \pi_{1}(t), \ldots, \pi_{C}(t)\right)$

$$
\frac{d}{d t} \pi_{j}(t)=\sum_{i=0}^{C} \pi_{i}(t) a_{i j}, j=0,1, \ldots, C .
$$

The system of differential equations (18) can be written as a matrix differential equation: $\dot{\pi}=\pi$ A . Having an initial distribution $\underline{\pi}_{0}$, the state probability vector $\pi(t)$ for future time instants $t, t>0$, is obtained as: $\underline{\pi}(t)=\underline{\pi}_{0} e^{A t}$, where $e^{A t}$ is the matrix exponential function of $A t$, and it is defined as $\left(I+A t+1 / 2(A t)^{2}+1 / 6(A t)^{3}+\ldots\right)$.

Equation (18) and its solution suggest that the expected actual link load may be roughly approximated by a scalar exponential function, decaying from the initial value $u_{0}$ to the asymptotic value $u_{\infty}$. The asymptotic value can be obtained from the steady state distribution $q$ as $u_{\infty}=q \cdot(0,1, \ldots, C)$. From the point of view of the well known theory of linear differential equations, the decay constant $\alpha$ of the exponential approximation should be in the range of the real component of the eigenvalue of $A$ with the smallest absolute value, which is not equal to zero. For practical implementation, the value $\alpha$ was computed in analogy to the link load estimation in [6] as the sum of transition rates towards higher occupancy states $u$ minus the transition rates toward lower states $u$, averaged over two points $u=C$ and $u=1 / 2\left(C-u_{\infty}\right)$. The link load estimation $u(t)$ is then obtained as follows $(\alpha<0)$ :

$$
u(t)=u_{\infty}+\left(u_{0}-u_{\infty}\right) e^{\alpha t}
$$




\section{ROUTING SCHEMES}

\subsection{DR/ATM}

DR/ATM uses a routing architecture which was developed for the IN/DR scheme [6] and further adapted to ATM networks. It is based on regional routing servers, the routing control processors (RCPs). The ATM network is subdivided into routing domains. Each domain contains one RCP, and all ATM nodes within the domain are connected to the respective RCP. This concept of routing domains allows to achieve a scalability of the DR/ATM scheme to very large networks.

For each call, the first routing attempt is made on the default route, in strong mesh networks, or, in weak mesh networks, on a randomly chosen minimum-hop route. If the first routing attempt fails, the connection is cranked back to the source node, which sends a "routing query" to the RCP in its domain. The routing decision is computed within the RCP based on the ATM-FLR link cost function and on the link load estimations. The alternative route with the lowest path cost is chosen, if the path cost does not exceed a threshold, otherwise the call is rejected. The offered load value used for the link load estimations is determined from the frequency of routing queries. The routing decision is then sent from the RCP to the ATM node, which routes the call. In networks of large geographical extent, RCPs exchange data related to their domain with other RCPs periodically, via satellite broadcast.

\subsection{LLP}

The least loaded path routing (LLP) examines for all paths, the minimum free capacity of included links, i. e. the "bottleneck" of each path. The path with the largest bottleneck capacity is selected. Alternative paths are allowed only if the free capacity is greater than a certain threshold (trunk reservation). In the simulations shown in Section 4, LLP is implemented as follows:

- The first routing attempt is made on the default path.

- If the first attempt fails, the least loaded alternative path is selected

- Trunk reservation is applied

The actual link load is either derived from the link load estimation scheme or the exact link load is used. 


\subsection{Minimum-Hop Routing}

Minimum-hop routing selects a route with the minimum number of hops, among the available routes, by means of the Dijkstra algorithm. If all minimum-hop routes are blocked, a route with the next higher number of hops is selected. If several equivalent minimum-hop routes are found, one of these routes is selected randomly. In Section 4, the link load for minimumhop routing is known from link state flooding or no link state knowledge is assumed.

\subsection{Minimum-Cost Routing}

For minimum-cost routing, the route with the lowest total ATM-FLR path cost is chosen. The total path cost is computed as the sum of the actual link cost values for links belonging to the path. The actual links cost values are determined from the ATM-FLR link cost function and from the actual link load, as obtained from link state flooding. If the minimum path cost exceeds a path cost threshold, the connection is rejected.

\section{SIMULATION}

\subsection{Network Models}

Three model networks are considered for simulations, a full mesh, a strong mesh and a square mesh ATM network, each consisting of twenty nodes. The link capacities range from 50 to $100 \mathrm{Mbps}$, in the full and strong mesh network. In the square mesh network, all links have 100 Mbps capacity. The total numbers of (unidirectional) links are 380 in the full mesh, 280 in the strong mesh and 62 in the square mesh network. A total of 27 call types with sustainable cell rate (SCR) values ranging from $64 \mathrm{kbps}$ to 4.5 Mbps, the average holding times were between 44 and 740 seconds. Complete fairness is applied, i.e. a call is accepted only if a call requesting the largest SCR value could be accepted instead. For all routing schemes only one alternative routing attempt is allowed at most for all connection attempts, except for non-alternate routing, where only default routes are allowed. 


\subsection{Simulation Results}

Figure 1 shows blocking curves obtained in simulation runs for the 20 node square mesh network. The offered load is shown in percentage of the nominal traffic. The nominal traffic corresponds to the $1 \%$ blocking level for non-alternate routing. The lowest blocking probabilities are achieved using exact knowledge of the actual link load (dotted lines). It can be seen that in this case the use of the ATM-FLR cost function for minimum-cost routing improves the blocking performance slightly as compared with LLP.

Comparing DR/ATM with a single domain and LLP using the link load estimation scheme (solid lines), it is seen, that DR/ATM achieves an impressive reduction of the blocking probability, especially in the low load region. At $0.1 \%$ blocking probability, DR/ATM allows to carry over 5\% more traffic than LLP.

Finally we consider the DR/ATM scheme with four routing domains (dashed line). The RCP-to-RCP update period is set to 30 seconds, i.e. each RCP sends its routing data, which is used for computing the link load estimation, every 30 seconds to the other three RCPs. It can be seen that only a moderate loss of blocking performance is obtained, as compared to DR/ATM, with a single routing domain. This shows that the concept of using routing domains may be successfully be applied in combination with the minimum cost routing and the link load estimation scheme.

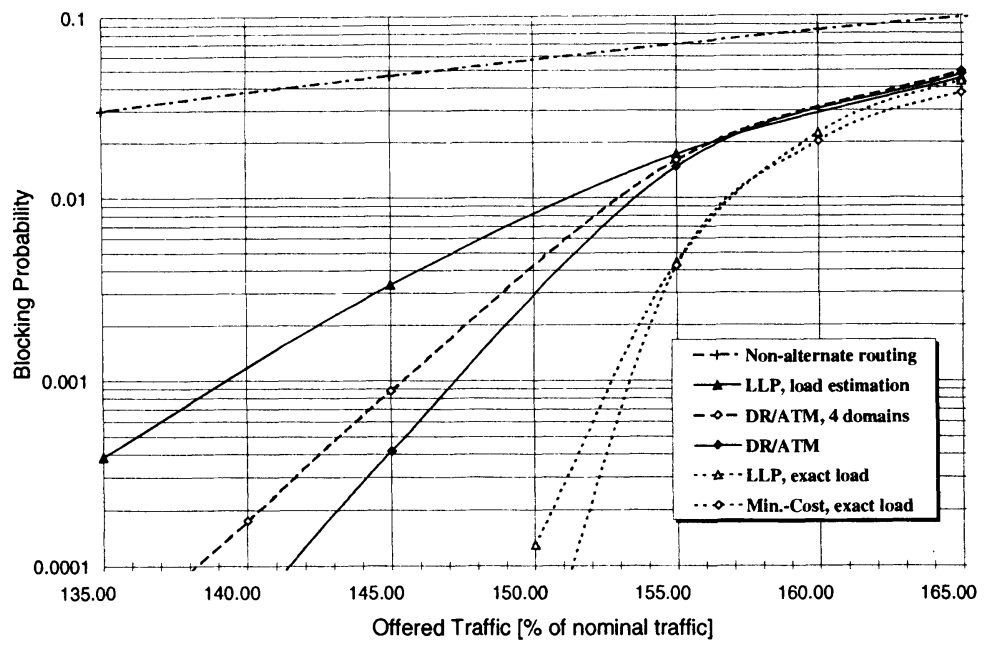

Figure 1: Blocking curves for the 20 node full mesh network.

The simulation results for the 20 node strong mesh network are shown in Figure 2. The uppermost line shows the blocking curve of non-alternate 
routing. The other two dotted lines represent the blocking curves of the LLP and ATM-FLR cost based routing with exact knowledge of link loads. It can be seen that by using the ATM-FLR cost function significantly lower blocking probabilities are obtained than with LLP.

As to the DR/ATM scheme and LLP using the link load estimation scheme, it can be seen that DR/ATM achieves lower blocking probabilities. However in the low load region, the improvement achieved by DR/ATM is less dramatic than for the full mesh network. A possible explanation for this effect is that in the link load estimation is less precise in the partial mesh case, particularly in the low load region. Nevertheless, at the $0.1 \%$ blocking level DR/ATM allows to carry $4.7 \%$ more traffic than LLP.

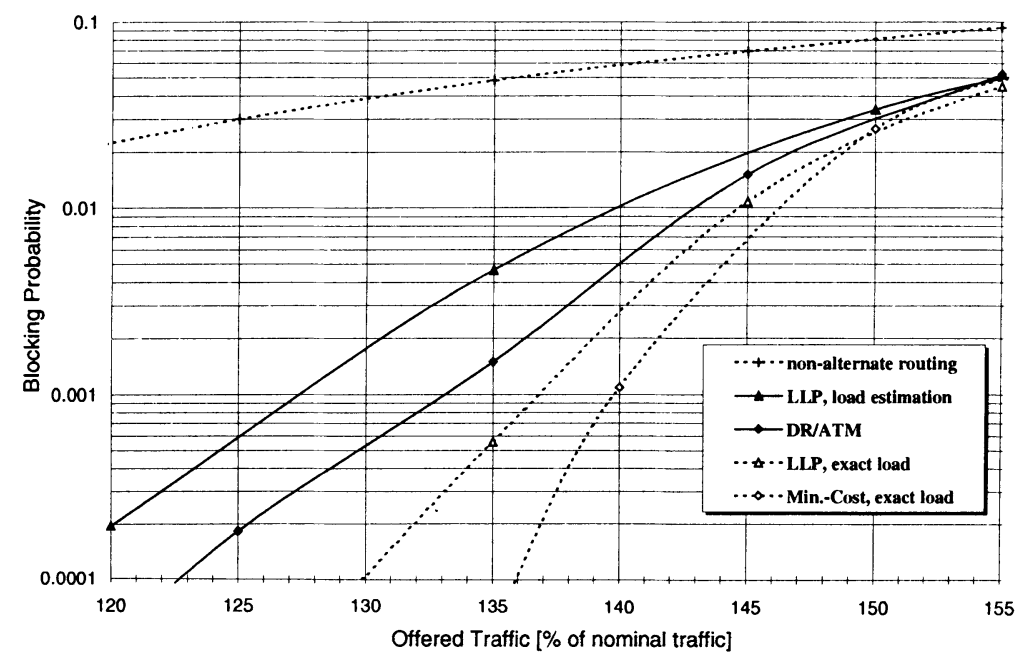

Figure 2: Blocking curves for the strong mesh 20 node network.

The blocking curves obtained for the 20 node square mesh network are represented in Figure 3. The highest blocking probabilities are achieved by minimum-hop routing without link state flooding (dotted curve). Lower blocking probabilities are obtained by minimum-hop routing with link state flooding and DR/ATM. The lowest blocking curve is obtained for minimumcost routing using the ATM-FLR link cost and link state flooding.

At high load levels, the blocking curves of minimum-hop routing with and without flooding assume similar values. Moreover, the blocking probabilities for the two ATM-FLR cost based schemes DR/ATM and minimum-cost routing are close to each other. The reason for this is that the link load estimation scheme used for DR/ATM is more precise in the high load region. 
As to the general routing performance, it is seen that at the $0.1 \%$ blocking level DR/ATM allows to carry $4.2 \%$ more traffic than minimum-hop routing with link state flooding. At the same blocking level minimum-cost routing with link state flooding allows to carry $8.3 \%$ more traffic than minimum-hop routing.

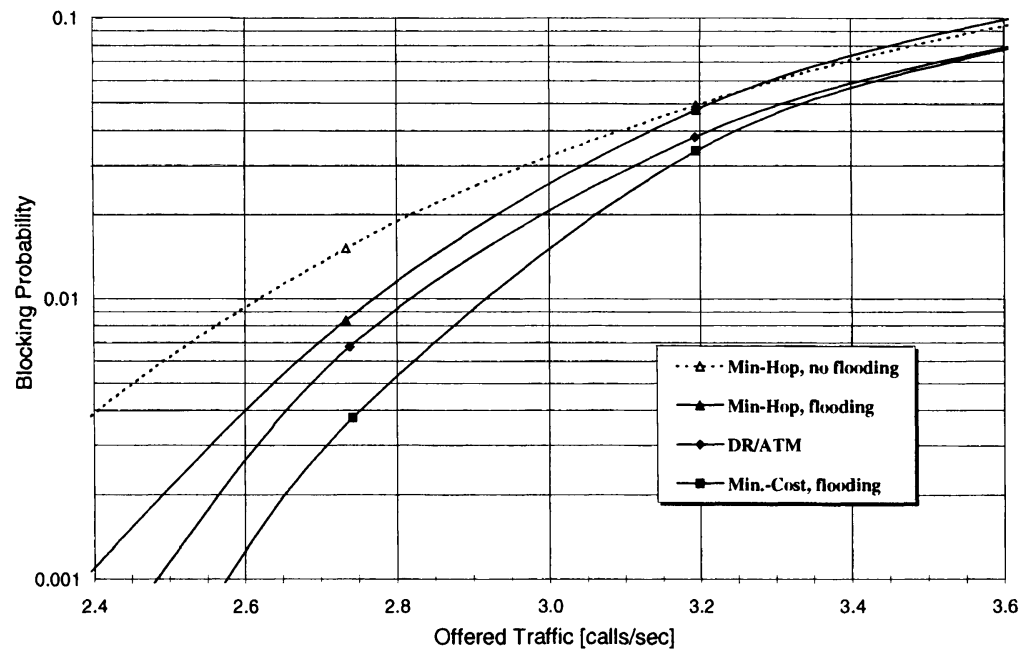

Figure 3: Blocking curves for the 20 node square mesh network.

\section{CONCLUSION}

Dynamic routing schemes have been shown in the past to reduce the call blocking probability in circuit-switched networks. In recent years several Markov decision theoretical approaches of various precision and complexity have been made to the routing problem for networks carrying multirate traffic. In this paper a new variant of defining a link cost function has been proposed. It has been shown that this link cost function can be computed by means of the so-called quotient formula. Moreover a method for obtaining offered link load values which can be used for the link cost computation in weak mesh networks has been introduced. The link cost function is independent of the type of the call to be routed. Thus possibly some routing performance is lost as compared to more precise routing schemes. However, it is seen as a benefit of the link cost function that only one actual link cost value per link needs to be stored and accessed in real time path computations.

The routing performance of the link cost function and the link load estimation method has been shown for a model routing scheme, DR/ATM. It 
is shown by simulation runs with different degrees of meshing that DR/ATM achieves an improved blocking performance, as compared with least loaded path routing and minimum-hop routing. Results regarding ATM-FLR link cost function and dynamic routing in networks running the Private Network Node Interface (PNNI) protocol can be found in [11]. A detailed description of the theoretical concepts and of the routing simulations can be found in [12].

\section{REFERENCES}

[1] K. R. Krishnan and T. J. Ott: „Forward-Looking Routing: A New State-Dependent Routing Scheme", ITC-12, pp. 1026-1031, 1989.

[2] R. Hwang, J. Kurose and D.Towsley: „State Dependent Routing for Multirate Loss Networks“, IEEE Globecom ‘92, vol.1, pp. 565-570, Orlando, FL, USA, Dec. 1992.

[3] A. Kolarov, J. Hui: „On Computing Markov Decision Theory-Based Cost for Routing in Circuit-Switched Broadband Networks “, Journal of Network and Systems Management, Vol. 3, No. 4, 1995.

[4] Z. Dziong and L. Mason: "An Analysis of Near Optimal Call Admission and Routing Model for Multi-Service Loss Networks”, IEEE Infocom '92, 1992, Vol. 2, pp. 142-152.

[5] K.R. Krishnan and F. Huebner-Szabo de Bucs:"Admission Control for Multirate CircuitSwitched Traffic", ITC-15, 1997.

[6] L. Bella , F. Chummun, M. Conte, G. Fischer, and J. Rammer,.: "Performance Evaluation of Dynamic Routing Based on the Use of Satellites and Intelligent Networks", in Wireless Networks 4, 167-180 (1998).

[7] H. Akimaru and K. Kawashima, Teletraffic Theory and Applications, Telecommunication Networks and Computer Systems, Springer-Verlag, 1993.

[8] R. B. Cooper, Introduction to Queueing Theory, North Holland, 1972.

[9] J. S. Kaufman, "Blocking in a Shared Resource Environment", IEEE Transaction on Communications, COM-29, Nr.10, pp. 1474-1481, October 1981.

[10] R. A. Howard: Dynamische Programmierung und Markov-Prozesse, (translation) 1965.

[11] M. Conte, J. Rammer, G.Fischer, N. Mersch, F. Chummun, L. Bella: "Simulation Study of Dynamic Cost-Based Routing in PNNI Networks, to appear in IEEE ATM Workshop 2000, June 2000.

[12] M. Conte: "Dynamic Routing Schemes for ATM Networks", Dissertation, TU Wien, to be published.

\section{ACKNOWLEDGMENT}

The work reported here was done under European Space Agency (ESA) contract. The contributions of F. Chummun, L. Bella, G. Fischer and J. Rammer are acknowledged. The author would like to thank J. Rammer for a critical reading of the manuscript. 